\title{
Near-infrared integral-field spectroscopy of violent starburst environments
}

\author{
Richard de Grijs \\ Department of Physics \& Astronomy, The University of Sheffield, Hicks Building, \\ Hounsfield Road, Sheffield S3 7RH, UK
}

\begin{abstract}
Near-infrared (NIR) integral-field spectroscopy (IFS) of violent starburst environments at high spatial (and spectral) resolution has the potential to revolutionise our ideas regarding the local interactions between the newly-formed massive stars and the interstellar medium (ISM) of their host galaxies. To illustrate this point, I present NIR IFS analysis of the central starburst region of NGC 1140, obtained with CIRPASS on Gemini-South. While strong [FeII] emission is found throughout the galaxy, higher-order Brackett emission is predominantly associated with the northern starburst region. Based on the spatial distributions of the [FeII] versus Brackett line emission, I conclude that a galaxy-wide starburst was induced several $\times 10^{7} \mathrm{yr}$ ago, with more recent starburst activity concentrated around the northern starburst region. I look forward and discuss the exciting prospects that IFS at higher spatial (and spectral) resolution will allow us trace (i) the massive outflows ("superwinds") expected to originate in the dense, young massive star clusters commonly found in intense starburst environments, and (ii) their impact on the galaxy's ISM.
\end{abstract}

Key words: galaxies: evolution, galaxies: starburst, infrared: galaxies PACS: 95.55.Qf, 98.58.Hf, 98.58.Mj, 98.62.Ai

\section{NGC 1140: A case for galactic cannibalism?}

The southern starburst dwarf galaxy NGC 1140 is remarkable in the number of compact, young, massive star clusters (YMCs) that it has formed recently in the small volume of its bright emission-line core. Based on HI and optical observations [e.g., Hunter, O'Connell \& Gallagher 1994a [hereafter H94a], de Grijs et al. 2004], and comparison with models (Hunter, van Woerden \& Gallagher 1994b, hereafter H94b), it exhibits the characteristics of a recent merger event, which has presumably induced the YMC formation in its centre, and which is currently in its final stages. The galaxy is unusually bright for its 
dwarf (or amorphous) morphological type, and at faint Hi and optical light levels it shows multiple, misaligned shell-like structures (H94a; see also Fig. 1). These are reminiscent of the shells associated with some elliptical galaxies that are thought to be remnants of a past tidal encounter (e.g., Schweizer \& Seitzer 1988, Hernquist \& Spergel 1992).

The galaxy is also unusual in its content and overall morphology: its optical appearance is dominated by a supergiant HII region encompassing most of its centre, with an $\mathrm{H} \alpha$ luminosity far exceeding that of the giant HII region 30 Doradus in the Large Magellanic Cloud (H94b). Its gas fraction, gas-toluminosity ratio, and $\mathrm{HI}$ velocity dispersion are unusually high, and its $\mathrm{H} \alpha$ velocity profiles remarkably broad, by roughly an order of magnitude, for the mid-spiral type galaxy one would expect, based on its total mass (H94b).

In addition, the formation of compact YMCs seems to be a hallmark of intense episodes of recent, active star formation. Compact YMCs are therefore important as probes of their host galaxy's recent star-formation history, and also of its chemical evolution, the stellar initial mass function (IMF), and other physical characteristics in starbursts.

Although NGC 1140 has a small dwarf companion galaxy (which might suggest that gravitational interactions with this companion may have induced part of the recent active star formation), these observations led Hunter et al. (H94b) to conclude that the galaxy is most likely the product of the accretion of a low surface brightness gas-rich companion by a relatively normal mid-type spiral galaxy of roughly equal mass in the last $\sim 1 \mathrm{Gyr}$. This picture is consistent with the low metallicities and complex Hi morphology (including a warped disc) and velocity field.

\section{The promise of integral-field spectroscopy}

NGC 1140 is in many ways similar to the prototype starburst galaxy M82, in which we discovered a large population of $\sim 1$ Gyr-old YMCs (de Grijs et al. 2001, 2003). It does not, however, feature a minor-axis superwind as in M82.

The interplay between the YMCs and the galaxy's interstellar medium (ISM) is, as in most active starburst galaxies, highly complex. Unlike in M82, however, the average internal extinction appears to be moderate (see de Grijs et al. 2004 for an overview). Nevertheless, in order to (i) minimise the effects of residual internal extinction, and (ii) sample the highly complex starburst environment at the highest spatial and spectral resolution, we obtained near-infrared imaging spectroscopy with the Cambridge InfraRed Panoramic Survey Spectrograph (CIRPASS; Parry et al. 2000), which was deployed at Gemini-South 


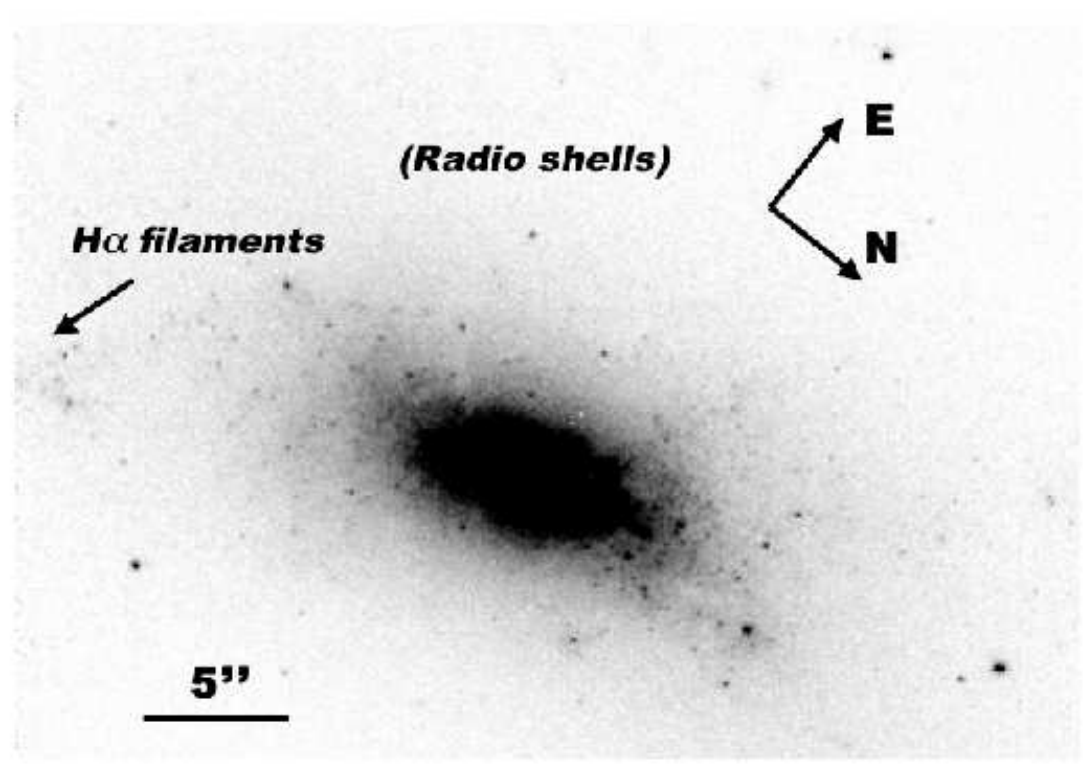

Fig. 1. NGC 1140 as seen with the Hubble Space Telescope through the F555W ( $V$-band) filter. The contrast has been chosen to show the low-level structures; the location of the radio shells identfied by Hunter et al. (H94a) is indicated, as is the direction in which $\mathrm{H} \alpha$ filaments extend (e.g., Jansen 2004).

as part of the instrument commissioning/demonstration science programme, carried out in Director's Discretionary Time (on UT 6 August 2002).

We aimed at obtaining significantly more accurate age estimates for the YMCs than broad-band Hubble Space Telescope observations would allow us (cf. H94a, de Grijs et al. 2004), particularly for the more obscured YMCs. This would allow us to independently verify their tidal interaction-induced starburst 
origin (e.g. H94b). In addition, the two-dimensional spectroscopic sampling would - for the first time - allow us to assess the state and age of the disk stellar population and the ISM. Thus, we expected that our CIRPASS data would allow us to construct an "age map" of NGC 1140's central starburst region, which could then be compared with the dynamical models for the interaction-induced starburst (e.g. H94b).

CIRPASS is a NIR fibre-fed spectrograph, connected to a 490-element integralfield unit (IFU). The variable lenslet scale was set to 0.36 arcsec diameter, and the hexagonal lenslets are arranged in the IFU to survey an approximately rectangular area of $13.0 \times 4.7$ arcsec, which provides sufficient sampling of the unusually bright diffuse light in NGC 1140. A $400 l \mathrm{~mm}^{-1}$ grating was used, which produced a dispersion of $2.25 \AA$ pixel $^{-1}$ and a wavelength coverage of $1.45-1.67 \mu \mathrm{m}$, with a resolving power of $R \equiv(\lambda / \Delta \lambda)_{\mathrm{FWHM}}=3500$.

The observations were taken in non-photometric conditions, with a seeing of $\approx 1.0-1.5$ arcsec FWHM. We obtained three 40-minute exposures of the galaxy, and one 40-minute offset sky exposure. Although we had noticed that our last 40-minute exposure was affected by high winds (and therefore blurred), during the analysis stage we found that the guiding of one of the other exposures had experienced problems as well due to wind shake. Thus, the CIRPASS results discussed here are based on the single 40-minute exposure obtained under the best available conditions.

\section{Propagation of star-formation activity?}

The wavelength range from $1.45-1.67 \mu \mathrm{m}$ covers a number of emission lines expected to be strong in active starburst regions. We identified four strong emission lines in a significant fraction of the lenslets covering the NGC 1140 starburst, three of which were located in spectral regions well away from the $\mathrm{OH}$ sky lines, and clearly resolved. In addition to strong [FeII] emission (at $\left.\lambda_{\text {rest }}=1.644 \mu \mathrm{m}\right)$, we also detect the hydrogen lines $\operatorname{Br} 12-4\left(\lambda_{\text {rest }}=1.641 \mu \mathrm{m}\right)$, $\operatorname{Br} 13-4\left(\lambda_{\text {rest }}=1.611 \mu \mathrm{m}\right.$; although significantly affected by a nearby strong sky line) and $\operatorname{Br} 14-4\left(\lambda_{\text {rest }}=1.588 \mu \mathrm{m}\right)$. We also detect a weak absorption feature at the wavelength range expected for the $\mathrm{CO}(6,3)$ band head, in the most active starburst regions (at $\lambda \simeq 1.627 \mu \mathrm{m}$, after correcting for the galaxy's redshift; see de Grijs et al. 2004). Its signal-to-noise (S/N) ratio was not sufficient for reliable absorption-line studies, however.

In Fig. 2 we show the flux distribution of the [FeII], Br 12-4 and $\operatorname{Br} 14-4$ emission lines across NGC 1140, compared to the Hubble Space Telescope $V$-band morphology. While [FeII] emission is found throughout the galaxy, the emission from all of the higher-order Brackett lines (including $\mathrm{Br} 12-4$, 


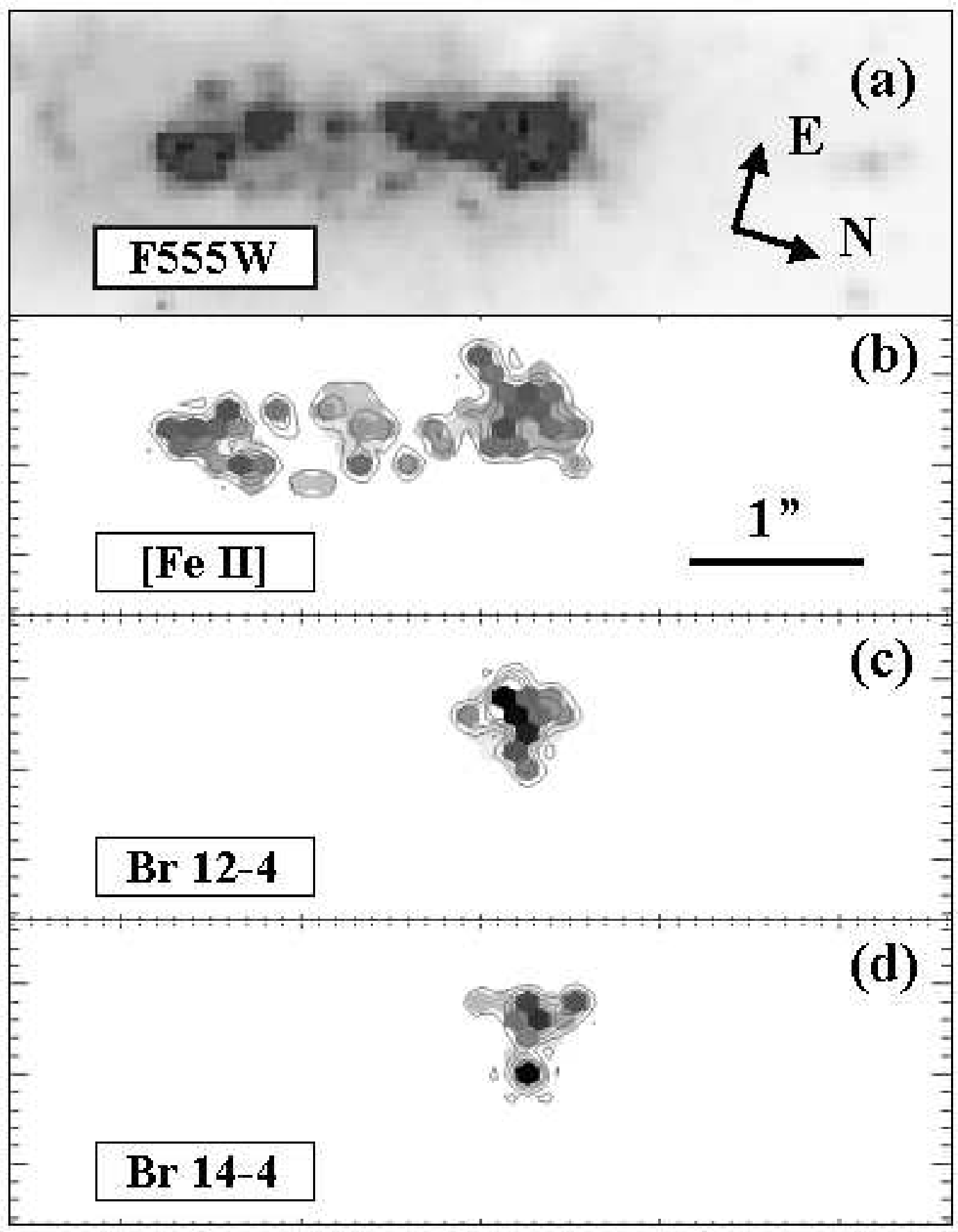

Fig. 2. Maps of (a) the Hubble Space Telescope $V$-band continuum flux distribution, (b) the [FeII] emission, (c) the $\mathrm{Br} 12-4$, and (d) the Br 14-4 emission-line morphologies of NGC 1140 (from de Grijs et al. 2004). The CIRPASS data show both the flux levels in the individual fibre lenses (above $\sim 3 \sigma$, where $\sigma$ is the noise in the continuum level of the spectra near the respective emission lines), as well as smoothed contours of the same data. 
Br 13-4 and $\mathrm{Br} 14-4)$ is found predominantly associated with the northern starburst region, NGC 1140-N. Although the observing conditions under which the CIRPASS data were obtained were non-photometric, the fact that we observe all wavelengths simultaneously over the entire two-dimensional field of view enables us to say with confidence that this different spatial distribution is real. This is one of the major advantages of using IFUs.

The [FeII] line on the one hand, and the Brackett 12-4 and 14-4 lines on the other originate in physically distinct processes, each governed by their unique time-scale(s). [FeII] $\lambda 1.64 \mu \mathrm{m}$ line emission originates through two different mechanisms. Strong, compact [FeII] emission originates in partially ionised zones or shock-excited gas produced by supernovae (SNe). More diffuse [FeII] emission seems to be associated with galactic superwinds. In starburst galaxies, compact [FeII] emission is thought to arise from discrete SN remnants (e.g., Oliva, Moorwood \& Danziger 1989, Lumsden \& Puxley 1995, Vanzi \& Rieke 1997, Morel, Doyon \& St-Louis 2002, Alonso-Herrero et al. 2003). Its excitation requires both the destruction of dust grains (which contain a large fraction of the interstellar iron) and large transition zones between HiI regions and regions of neutral hydrogen, in association with a hard ionising source (so that the electron temperature is sufficiently high to excite the forbidden [FeII] transitions; e.g., Oliva et al. 1989). Such spatial scales therefore imply that thermal shock excitation is favoured (Oliva et al. 1989, Vanzi \& Rieke 1997).

Brackett lines, on the other hand, are formed by recombination in ionised gas associated with sources having a strong Lyman continuum, such as HII regions created by young, massive stars. The strong Brackett line emission will fade effectively after $\sim 8 \mathrm{Myr}$, while the [FeII] emission remains observable significantly longer; the $[\mathrm{FeII}] /$ Brackett line ratio is therefore age dependent.

This difference in origin is quantitatively supported by our line strength measurements. While both the composite $\mathrm{Br} 12-4$ and $\mathrm{Br} 14-4$ emission lines in NGC 1140-N are unresolved, the [FeII] lines are clearly resolved. We measure intrinsic FWHMs (i.e., corrected for the widths of unresolved lines, 3.8A) of $5.9 \AA$ and $4.2 \AA$ in starburst regions NGC $1140-\mathrm{S}$ (the southern starburst) and $\mathrm{N}$, respectively. This implies that the velocity dispersions are $\sim 75$ and $\sim 110$ $\mathrm{km} \mathrm{s}^{-1}$ in NGC 1140-S and N, respectively. This is consistent with the scenario that we are seeing outflows in the [FeII] emission, most likely driven by the combination of SNe and stellar winds associated with the starburst.

The last SNe in a quenched starburst region occur at a time comparable to the longest lifetime of a SN progenitor after the end of the starburst activity, 35 - 55 Myr (see de Grijs et al 2004 for a discussion). For NGC 1140, we conclude therefore that the presence of strong [FeII] emission throughout the central galaxy implies that most (but perhaps not all, allowing for the presence of unresolved [FeII] emission outside the currently active starburst regions) of 
the recent star-formation activity was induced some $35-55 \mathrm{Myr}$ ago.

Since the Br 12-4 and Br 14-4 emission, concentrated around NGC 1140-N is associated with recombination processes in younger HiI regions, this implies that the most recent starburst event has transpired in this more confined area of the NGC 1140 disc. Thus, based on the [FeII] versus Brackett line emission, we conclude that a galaxy-wide starburst was induced several $\times 10^{7}$ yr ago, with more recent starburst activity concentrated around the northern starburst region, NGC 1140-N.

\section{Higher resolution: Spatial vs. spectral resolution improvements}

Although our poor-quality CIRPASS observations provided us with interesting insights into the galaxy's global recent star-formation history, they did not allow us to probe the physically interesting parsec scales, nor to resolve the individual YMCs (for which a seeing $<0.7$ arcsec FWHM is required). At a spatial resolution of $\lesssim 0.5-0.7$ arcsec, we would be able to (just) separate the stellar and gaseous components in the active starburst regions, and thus place the current starburst in the context of the evolution of this starburst galaxy as a whole.

Based on higher spatial resolution data, which are increasingly likely to become available in the near future - in particular in view of worldwide efforts to provide high-quality adaptive optics-enabled acquisition of scientific data I expect to be able to zoom in into the galaxy's active starburst, and distinguish between the YMCs and the intercluster medium, and thus follow cluster winds and other outflows triggered by the ongoing starburst. The origin of the ubiquitous outflows and feedback processes in active starburst galaxies is as yet a key open question in this very active field, the answers to which will most likely be provided by adaptive optics-assisted IFS.

Secondly, the $R \sim 3500$ spectral resolution of CIRPASS proved to be just sufficient to determine overall radial velocities to within several tens of $\mathrm{km}$ $\mathrm{s}^{-1}$ for entire starburst regions (de Grijs et al. 2004), but insufficient to determine the individual YMC velocities, nor those of the gaseous ISM. While the $R \sim 3500$ resolution would in principle have been sufficient for our YMC age determinations, at higher spectral resolution (e.g., $R \gtrsim 6000$ ) we expect to more easily resolve the stellar and cluster winds and outflows, as well as the general kinematics of the underlying stellar disk.

Finally, perhaps the simplest approach to improving the scientific results in this contribution is to obtain higher- $\mathrm{S} / \mathrm{N}$ observations, and use these to redo the analysis. For instance, the $\mathrm{CO}$ band head in the $H$ band is present, but 
in our CIRPASS data of insufficient quality to measure its equivalent width robustly nor reliably, due to the poor quality and low $\mathrm{S} / \mathrm{N}$ ratio of the observations at hand. I point out, however, that the $\mathrm{CO}$ band head features are significantly stronger in both the $J$ and $K$ bands, thus suggesting that these might be more appropriate wavelength ranges to target in studies of the associated red supergiant content of starburst galaxies.

Studies of starburst galaxies in the local Universe, particularly those involved in and/or triggered by galaxy interactions, provide key diagnostics in terms of the resulting time-scales of the star-formation activity and of the interaction history. Both are essential ingredients in our quest to understand the details of galaxy evolution as such. Examples from the local Universe, which we can study in great detail, have therefore the potential to provide key insights into galaxy evolution at higher redshifts, where galaxy interactions and starburst events were much more common than at the present, relatively quiescent epoch.

\section{Acknowledgements}

I am very grateful to Linda Smith, Rob Sharp, Jay Gallagher, and the CIRPASS team at the Institute of Astronomy in Cambridge, for their input and assistance during this project. Without them, this work could not have been completed successfully in a timely manner.

\section{References}

[] Alonso-Herrero A., Rieke G.H., Rieke M.J., Kelly D.M., 2003, AJ, 125, 1210

[] de Grijs R., O'Connell R.W., Gallagher J.S. III, 2001, AJ, 121, 768

[] de Grijs R., Bastian N., Lamers H.J.G.L.M., 2003, MNRAS, 340, 197

[] de Grijs R., Smith L.J., Bunker A., Sharp R.G., Gallagher J.S. III, Lançon A., O'Connell R.W., Parry I.R., 2004, MNRAS, 352, 263

[] Hernquist L., Spergel D.N., 1992, ApJ, 399, L117

[] Hunter D.A., O'Connell R.W., Gallagher J.S. III, 1994a, AJ, 108, 84 (H94a)

[] Hunter D.A., van Woerden H., Gallagher J.S. III, 1994b, ApJS, 91, 79 (H94b)

[] Jansen R.A., 2004, in: Penetrating Bars Through Masks of Cosmic Dust: The Hubble Tuning Fork Strikes a New Note, Block D.L., Freeman K.C., eds., ASSL Vol. 319, Spinger: Dordrecht, p. 423

[] Lumsden S.L., Puxley P.J., 1995, MNRAS, 276, 723

[] Morel T., Doyon R., St-Louis N., 2002, MNRAS, 329, 398

[] Oliva E., Moorwood A.F.M., Danziger I.J., 1989, A\&A, 214, 307 
[] Parry I.R., et al., SPIE, 4008, 1093

[] Schweizer F., Seitzer P., 1988, ApJ, 328, 88

[] Vanzi L., Rieke G.H., 1997, ApJ, 479, 694 